\title{
ERRATUM
}

\section{Erratum to: Altered regional homogeneity in Posttraumatic Stress Disorder: a resting state functional magnetic resonance imaging study}

Yan Yin ${ }^{1,2}$, Changfeng Jin ${ }^{1}$, Lisa T. Eyler, ${ }^{4,5}$, Hua Jin ${ }^{4,6}$, Xiaolei Hu ${ }^{1,7}$, Lian Duan ${ }^{1}$, Huirong Zheng ${ }^{8}$, Bo Feng ${ }^{9}$, Xuanyin Huang ${ }^{10}$, Baoci Shan ${ }^{11}$, Qiyong Gong ${ }^{12}$, Lingjiang $\mathrm{Li}^{1,3}$

'Mental Health Institute, The Second Xiangya Hospital of Central South University, Changsha 410011, China

${ }^{2}$ Shenyang Gongan Hospital, Shenyang 110003, China

${ }^{3}$ Chinese University of Hong Kong, Hong Kong, China

${ }^{4}$ Department of Psychiatry, University of California-San Diego School of Medicine, San Diego, California, USA

${ }^{5}$ VA San Diego Mental IIIness Research Education and Clinical Center, San Diego, California, USA

${ }^{6} V A$ San Diego Healthcare System, San Diego, California, USA

China University of Mining and Technology, Xuzhou 221116, China

${ }^{8}$ Guangdong Mental Health Institute, Guangdong General Hospital, Guangdong Academy of Medical Sciences, Guangzhou 510120, China

'Mianzhu Psychiatric Hospital, Deyang 618200, China

${ }^{10}$ Mental Health Center of Sichuan Province, Mianyang 621000, China

"Institute of High Energy Physics, Chinese Academy of Sciences, Beijing 100049, China

${ }^{12} H u a x i$ MR Research Center, Department of Radiology, West China Hospital of Sichuan University, Chengdu 610041, China

(C) Shanghai Institutes for Biological Sciences, CAS and Springer-Verlag Berlin Heidelberg 2013

Erratum to: Neurosci Bull October 1, 2012, 28(5): 541-549

DOI: $10.1007 / \mathrm{s} 12264-012-1261-3$

The original version of this article unfortunately contained a mistake. The presentation of " 3100 survivors" in the 3 rd line from bottom in right column on page 542 is incorrect. The corrected should be "4200 survivors".

The online version of the original article can be found at:

http://dx.doi.org/10.1007/s12264-012-1261-3

\footnotetext{
Corresponding author: Lingjiang $\mathrm{Li}$

Tel: +86-731-85550413; Fax: +86-731-85360086

E-mail: Ilj2920@163.com
} 